\title{
The moderate magnetic field of the flare star Proxima Centauri
}

\author{
A. Reiners ${ }^{1, \star}$ and G. Basri ${ }^{2}$ \\ 1 Universität Göttingen, Institut für Astrophysik, Friedrich-Hund-Platz 1, 37077 Göttingen, Germany \\ e-mail: Ansgar.Reiners@phys.uni-goettingen.de \\ 2 Astronomy Department, University of California, Berkeley, CA 94720, USA \\ e-mail: basri@berkeley.edu
}

Received 1 July 2008 / Accepted 21 August 2008

\section{ABSTRACT}

\begin{abstract}
We report moderate magnetic flux of $450 \mathrm{G}<B f<750 \mathrm{G}(3 \sigma)$ on the nearby M 5.5 flare star Proxima Centauri. A high resolution UVES spectrum was used to measure magnetic flux from Zeeman broadening in absorption lines of molecular FeH around $1 \mu \mathrm{m}$. The magnetic flux we find is relatively weak compared with classical strong flare stars, but so are Proxima's flaring rates and actual emission levels. We compare what is known about the rotation rate, Rossby number, and activity levels in this star to relations between these quantities that are being developed more generally for $\mathrm{M}$ dwarfs. We conclude that the magnetic flux is higher than the best estimates of the Rossby number from period measurements. On the other hand, the activity levels of Proxima Centauri are at the high end of what could be expected based on the measured field, but not so high as to exceed the natural scatter in these relations (other stars lie along this high envelope as well).
\end{abstract}

Key words. stars: activity - stars: late-type - stars: individual: Proxima Centauri - stars: magnetic fields stars: low-mass, brown dwarfs

\section{Introduction}

Proxima Centauri ( $\alpha$ Cen C, GJ 551 C, in the following Prox Cen), our closest stellar neighbor, has been known to be a flare star for more than 50 years (Thackeray 1950). Prox Cen is of particular importance for our understanding of very cool stars because we can observe it with very high precision. On Prox Cen, activity can be detected at very low levels, and great effort has been put into monitoring campaigns to investigate it. At a spectral type of M5.5, Prox Cen is probably completely convective. It shows persistent $\mathrm{H} \alpha$ emission and flaring, which may lead to the expectation of rapid rotation $\left(\gtrsim 3 \mathrm{~km} \mathrm{~s}^{-1}\right)$. However, the strength of quiescent activity in Prox Cen is relatively low compared with other active mid-M stars (Mathioudakis \& Doyle 1991; Patten 1994; Hawley et al. 1996; Bochanski et al. 2007), and the flare rate is as well (Walker 1981).

The relation between rotation and magnetic activity is well known in solar-type stars (e.g. Pizzolato et al. 2003). It is observed in very slowly rotating early-M dwarfs $(v \sin i \gtrsim$ $1 \mathrm{~km} \mathrm{~s}^{-1}$, Reiners 2007), too. Mohanty \& Basri (2003) found that the rotation-acitivity relation is valid in low-mass stars down to spectral class M9, at least to the extent that rapid rotators show activity saturation. In solar-type stars, chromospheric and coronal activity is believed to be due to magnetic fields generated through a magnetic dynamo, and Reiners \& Basri (2007) found that magnetic flux scales with $\mathrm{H} \alpha$ activity also in the late-M dwarfs. This all leads to the expectation that activity in a flare star like Prox Cen is due to strong magnetic fields generated through a magnetic dynamo that may be driven by rotation.

There has been considerable effort to determine the rotation period of Prox Cen. Benedict et al. (1998) searched for a rotation period in HST FGS data claiming a rotation period of

^ Emmy Noether Fellow.
$P=83.5 \mathrm{~d}$, and Guinan \& Morgan (1996) report a period of $31.5 \mathrm{~d}$ from IUE data. From the intensity of $\mathrm{Mg}$ II emission lines in the same IUE data, Doyle (1987) predicted a rotation period of around $51 \mathrm{~d}$ according to the rotation-activity relation. However, Doyle (1987) also predicted a rotation period of $27 \mathrm{~d}$ for UV Cet. UV Cet has a projected rotational velocity of $v \sin i=32.5 \mathrm{~km} \mathrm{~s}^{-1}$ (Mohanty \& Basri 2003), i.e. a period of less than about half a day, which means that an estimate for the rotational period from $\mathrm{Mg}$ II data is probably not very useful in mid-M dwarfs. Recently, Kiraga \& Stępień (2007) reported a rotation period of $82.5 \mathrm{~d}$ for Prox Cen, very close to the $83.5 \mathrm{~d}$ period reported by Benedict et al. (1998). Altogether, there is evidence that $\sim 83 \mathrm{~d}$ is the true rotational period of Prox Cen.

The rotation velocity of Prox Cen is probably rather low, but still it shows persistent flaring activity. Although its flare rate is below those of the very active flare stars like CN Leo, it is still an active star and strong magnetic flux might be expected. In this paper we present the first direct measurement of the magnetic flux of Prox Cen.

\section{Data}

We retrieved our data from the ESO/ST-ECF Science Archive. An observation of Prox Cen was taken with UVES at VLT's UT2 on April 18, 2006. The data is described in more detail in Kurosawa et al. (2006); they were taken with the red arm centered at $860 \mathrm{~nm}$ providing wavelength coverage from 6700 $10250 \AA$ A. At a slit length of $1^{\prime \prime}$ the resolving power is $R \approx$ 40000 . Pipeline-reduced data were taken from the archive and used for our analysis. Standard reduction steps involve bias and background subtraction, flat-fielding, optimum extraction, and order-merging. 
The red setting used in this exposure covers the absorption band of the molecule $\mathrm{FeH}$, which we use for our determination of the magnetic flux in the following. After an exposure time of $100 \mathrm{~s}$, a signal-to-noise ratio of about 60 is achieved in this spectral range. Unfortunately, $\mathrm{H} \alpha$ is not covered by this instrumental setup.

\section{Method}

The method we employ to measure the magnetic flux in Prox Cen was introduced by us in Reiners \& Basri (2006) and after that applied to a sample of M 2-M9 dwarfs in Reiners \& Basri (2007). Here, we give a brief overview of the method and refer to these two papers for a more detailed description.

The absorption band of $\mathrm{FeH}$ contains a forest of strong, well isolated lines of which some are sensitive to the Zeeman effect caused by magnetic fields, while others are not. In a relatively small spectral range, we find spectral lines of the same ro-vibrational transition that react differently to the presence of a magnetic field. The direct simulation of the Zeeman effect in $\mathrm{FeH}$ calculating polarized radiative transfer is still hampered by the lack of Landé factors. Instead, we choose a more empirical approach: we observed two spectra of early-M dwarfs for which magnetic field measurements from atomic absorption lines exist. One of them shows no signs of magnetic fields (and no activity; GJ 1002), for the other a total magnetic flux of $B f \sim 3.9 \mathrm{kG}$ was measured (Gl 873, Johns-Krull \& Valenti 2000). The strategy we follow to measure the magnetic flux in Prox Cen is the following. We apply an optical-depth scaling to the two reference spectra so that the strength of the $\mathrm{FeH}$ band matches the strength of $\mathrm{FeH}$ absorption in Prox Cen. We can now use the magnetically insensitive lines to fix the rotational velocity, and the magnetically sensitive lines to adjust for the magnetic flux. This is done by interpolating the two template spectra (between zero magnetic flux and $B f=3.9 \mathrm{kG}$ ) in order to achieve the best fit to the spectrum of Prox Cen. Hereby, we assume that the Zeeman broadening is linear in $B f$ and that the distribution of magnetic field strength in Prox Cen is similar to the distribution in the magnetic reference star.

\section{Results}

Before the magnetic flux can be measured from the interpolation of the two reference spectra, the latter were adjusted for line depth and rotational broadening. The strength of the $\mathrm{FeH}$ band is about $85 \%$ of the depth seen in GJ $1002(a=1.2$, see Reiners \& Basri 2007); this is consistent with other old M5.5 dwarfs. The magnetically insensitive lines in Prox Cen show no extra broadening with respect to the template spectra, which implies that the projected rotational velocity is below the detection limit of $\approx 3 \mathrm{~km} \mathrm{~s}^{-1}$. This comes as no surprise, because several long rotational periods were reported from photometric observations. However, as we explained in Sect. 1, there is still some uncertainty about the rotation of Prox Cen, and to our knowledge no measurement of $v \sin i$ was reported for Prox Cen before.

In Fig. 1, we show the spectrum of Prox Cen (artificially broadened to match the spectral resolution of the template spectra) together with the reference spectra of GJ 1002 and Gl 873 scaled in optical depth to match the line strengths of Prox Cen. We searched for the best fit of the interpolation between the two reference spectra and the spectrum of Prox Cen calculating the summed quadratic difference $\chi^{2}$. The fit was calculated in three wavelength ranges, 9938.0-9943.5 A., 9947.3-9955.5 and 9971.5-9981.0 A; 901 pixels are used altogether. All three regions are shown in Fig. 1. The best fit is achieved for a ratio between our spectra with strong magnetic flux to zero flux of $15: 85$, i.e. $B f=0.15 \times 3.9 \mathrm{kG} \approx 600 \mathrm{G}$. With an SNR of 60 , we achieve a reduced chi-square of $\chi_{v}^{2}=0.86$ indicating a somewhat underestimated $\operatorname{SNR}\left(\chi_{\min }^{2}=775\right)$.

The fit quality in terms of $\chi^{2}$ is shown as a function of magnetic flux $B f$ in Fig. 2. We compute a formal $3 \sigma$ uncertainty in $B f$ by searching the range of $B f$ for which $\chi^{2}<\chi_{\min }^{2}+9$ while varying all other parameters (including rotational broadening). The formal result of this exercise is:

$B f=600 \mathrm{G} \pm 150 \mathrm{G}(3 \sigma)$.

There is some degeneracy between the derived magnetic flux and the rotational broadening $v \sin i$. In principle, we could use the magnetically insensitive lines to determine the broadening that applies to all spectral lines, i.e., residual instrumental broadening or $v \sin i$. With this information, we could then determine magnetic broadening using the magnetically sensitive lines. This approach has two caveats:

I) lacking molecular Landé factors, we cannot entirely trust the assumption that magnetically "insensitive" lines show absolutely no effect of magnetic fields - slight broadening due to magnetic fields may occur even in those lines;

II) using subsets of the spectrum would lead to different estimators of fit quality that are not necessarily comparable. This means that the parameters providing high fit quality in the magnetically insensitive lines may not lead to a good fit in the magnetically sensitive lines if only the magnetic flux is changed. This can only be avoided if all lines are used simultaneously. Thus, we prefer to always compute our $\chi^{2}$ values from the full spectral range.

In order to investigate the crosstalk between $B f$ and $v \sin i$ (or any instrumental broadening), we provide the landscape of $\chi^{2}$ as a function of overall broadening (e.g., $v \sin i$ ) and $B f$ in Fig. 3 This landscape shows the clear minimum in $\chi^{2}$ around $B f \approx 600 \mathrm{G}$. The minimum is also somewhat dependent on the choice of $v \sin i$. Interestingly, very slow values of " $v \sin i$ " are excluded. As mentioned above, the detection limit of overall line broadening at the spectral resolution of our data is of the order of $3 \mathrm{~km} \mathrm{~s}^{-1}$, i.e. anything below $3 \mathrm{~km} \mathrm{~s}^{-1}$ is not necessarily due to rotation, but is within the uncertainties from instrumental broadening. In other words, the fact that we require a broadening of the order of $2 \mathrm{~km} \mathrm{~s}^{-1}$ to fit the data does not imply that Prox Cen is rotating at this level, because at the given resolution a formal result of $v \sin i=2 \mathrm{~km} \mathrm{~s}^{-1}$ is fully consistent with zero rotation. On the other hand, the detection of the magnetic flux $B f$ comes from a differential analysis comparing lines of different magnetic sensitivity. Thus, no absolute lower limit of detectability (other than the calculated uncertainty that also depends on spectral resolution) applies.

\section{Discussion}

\subsection{Activity and magnetic flux}

Active M dwarfs typically exhibit magnetic flux of a few $\mathrm{kG}$ (Reiners \& Basri 2007). However, the magnetic flux of Prox Cen is substantially lower than $1 \mathrm{kG}$ although it clearly is an "active" star. Does Prox Cen have particularly weak magnetism given its level of magnetic activity? 

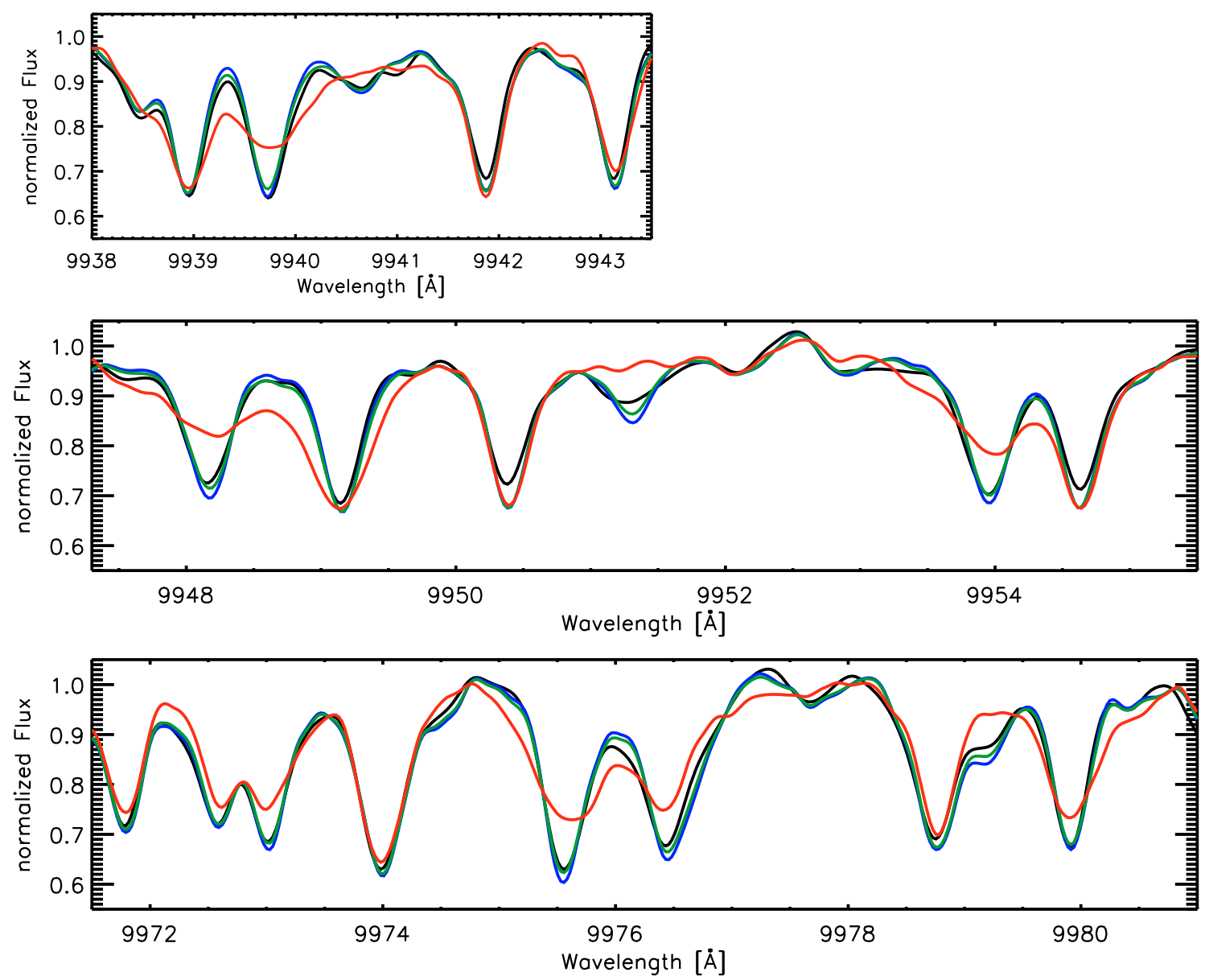

Fig. 1. Data of Prox Cen in the wavelength regions used for the fit. Black: data of Prox Cen; red: spectrum of a star with strong magnetic flux $(3.9 \mathrm{kG})$ scaled and artificially broadened to match the spectrum of Prox Cen; blue: as the red line but for a star with no magnetic flux; green: best fit linear interpolation between the case of very strong and no magnetic flux (at a ratio of $\sim 15: 85$, i.e. $\approx 0.6 \mathrm{kG}$ ).

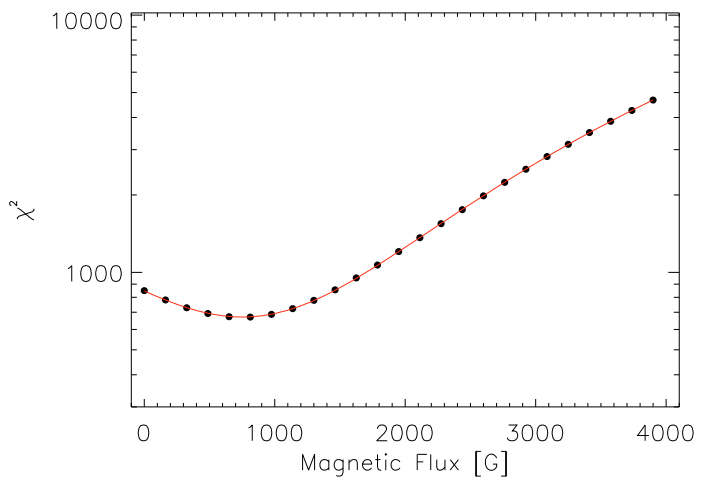

Fig. 2. Chisquare as a function of magnetic flux (see text).

The flare rate of Prox Cen is comparably low; Walker (1981) report 35 flares over $25.25 \mathrm{~h}$, which means it is less actively flaring than UV Cet, CN Leo, or EQ Peg. In particular, the flare rate of $\sim 1.4 \mathrm{~h}^{-1}$ found by Walker (1981) is only about $60 \%$ the flare rate of CN Leo found by Kunkel (1973) at comparable $U$-band sensitivity. This indicates that Prox Cen may be a flare star with relatively little activity.

Several measurements of the $\mathrm{H} \alpha$ emission of Prox Cen are reported in the literature. Mathioudakis \& Doyle (1991) measured an $\mathrm{H} \alpha$ equivalent width of $1.67 \AA$. Patten (1994) report a minimum of $3 \AA$, and $27 \AA$ during flare state, and

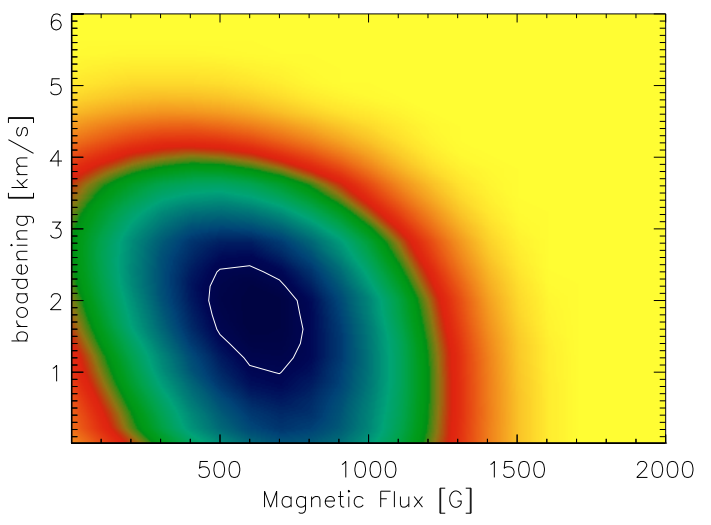

Fig. 3. Chisquare landscape as a function of $B f$ and $v \sin i$. The white line marks the region where $\chi^{2}<\chi_{\min }^{2}+9$.

Hawley et al. (1996) found an $\mathrm{H} \alpha$ emission line of $3.01 \AA$ equivalent width. The mean $\mathrm{H} \alpha$ equivalent width in active M5 dwarfs is $5.9 \pm 1.1 \AA$ (Bochanski et al. 2007, although one has to keep in mind that this is from data of lower resolution). The normalized $\mathrm{H} \alpha$ luminosities corresponding to $\mathrm{H} \alpha$ equivalent widths of $1.67 \AA$ and $3.0 \AA$ are $\log L_{\mathrm{H} \alpha} / L_{\mathrm{bol}}=-4.2$ and -4.0 , respectively (see Reiners \& Basri 2008a) ${ }^{1}$. According to

\footnotetext{
1 Hawley et al. (1996) calculate a value of $\log L_{\mathrm{H} \alpha} / L_{\mathrm{bol}}=-4.2$ for an equivalent width of $3.0 \AA$, i.e., 0.2 dex lower than our calibration.
} 
Mohanty \& Basri (2003), $\log L_{\mathrm{H} \alpha} / L_{\mathrm{bol}} \approx-4.2$ is the level where activity saturates in stars of spectral type M5.5-M8.5, i.e., activity in Prox Cen is barely in the regime of saturation. In fact, the lowest value of $\mathrm{H} \alpha$ emission (1.67 $\AA$ ) is comparable to $\mathrm{H} \alpha$ measured in GJ 1286 (1.50 ^) by Reiners \& Basri (2007). This M 5.5 star exhibits magnetic flux of $0.4 \pm 0.2 \mathrm{kG}(1 \sigma)$, which is very similar to Prox Cen (and GJ 1286 is a slow rotator).

Another proxy of magnetic activity is X-rays. X-ray emission of Prox Cen was measured with ROSAT and XMM: the X-ray flux measured with ROSAT is $\log L_{X}=27.2 \mathrm{erg} \mathrm{s}^{-1}$ (Hünsch et al. 1999). At $M_{\text {bol }}=11.98$ (Hawley et al. 1996) this means that the normalized X-ray luminosity is $\log L_{\mathrm{X}} / L_{\mathrm{bol}}=$ -3.5 . Newer observations from XMM are consistent with this result (see Schmitt \& Liefke 2004). Kiraga \& Stępień (2007) report a normalized X-ray luminosity of $\log L_{\mathrm{X}} / L_{\mathrm{bol}}=-3.8$. This value probably comes from the two longest ROSAT PSPC observations only, for which $\log L_{\mathrm{X}} \approx 26.9$ is provided by Schmitt \& Liefke (2004) (while $\log L_{X} \approx 27.4$ is given for the two XMM exposures).

For a mid-M dwarf, a normalized X-ray luminosity between $\log L_{\mathrm{X}} / L_{\mathrm{bol}}=-3.5$ and -3.8 is consistent with a normalized $\mathrm{H} \alpha$ luminosity between -3.7 and -4.2 (although the scatter is fairly large, see Reiners \& Basri 2007). This is consistent with $\mathrm{H} \alpha$ measurements (see above). Both X-ray and $\mathrm{H} \alpha$ emission put Prox Cen on the low end of active M dwarfs.

Reiners \& Basri (2007) investigated the relation between magnetic flux and $\mathrm{H} \alpha$ emission among $\mathrm{M}$ dwarfs. From their sample, a star of spectral class M5.5 with a normalized $\mathrm{H} \alpha$ luminosity of $\log L_{\mathrm{H} \alpha} / L_{\mathrm{bol}} \approx-4$ can be expected to have a magnetic flux level of the order of $B f \approx 1 \mathrm{kG}$. For $B f \approx 600 \mathrm{G}$, i.e. the magnetic flux found on Prox Cen, $\mathrm{H} \alpha$ emission of the order of $\log L_{\mathrm{H} \alpha} / L_{\mathrm{bol}}=-4.2$ can be expected. Thus, the magnetic flux estimated from the lowest X-ray and $\mathrm{H} \alpha$ detections is consistent with the magnetic flux measured here, while the emission seen in all X-ray and $\mathrm{H} \alpha$ data is scattered around a somewhat higher level. This leads to the conclusion that Prox Cen has somewhat higher activity than would be predicted from its magnetic field, but not an unreasonably higher amount, given that the scatter in all these relations is substantial.

\subsection{Rotation and Rossby number}

In M 5.5-M 8.5 stars, the rotation-activity relation saturates at a surface velocity of about $10 \mathrm{~km} \mathrm{~s}^{-1}$ (Mohanty \& Basri 2003). However, there are also slowly rotating mid-M stars that exhibit strong magnetic activity. While rapid rotators always seem to be active, the opposite is not true. Prox Cen shows no detectable Doppler broadening due to rotation; its projected rotation velocity is lower than $v \sin i \approx 3 \mathrm{~km} \mathrm{~s}^{-1}$. Reiners \& Basri (2007) found that all $\mathrm{M}$ dwarfs with magnetic flux $B f<1 \mathrm{kG}$ are slow rotators $\left(v \sin i<3 \mathrm{~km} \mathrm{~s}^{-1}\right.$ ). The low magnetic flux found for Prox Cen is consistent with slow rotation.

At a radius of $R=0.145 R_{\odot}$ (Ségransan et al. 2003), the spectroscopic limit on the rotation velocity translates into a projected rotation period of $P / \sin i>1 \mathrm{~d}$. There is growing evidence that the rotation period of Prox Cen is of the order of $80 \mathrm{~d}$ (Benedict et al. 1998; Kiraga \& Stępień 2007), which is consistent with the lack of Doppler broadening (although it is almost two orders of magnitude below the detection limit). A rotation period that long would imply an extremely low surface rotation velocity of $v=90 \mathrm{~m} \mathrm{~s}^{-1}$.

The turnover time of Prox Cen is in the range 70-100 d (Gilliland 1986). If the rotation period of Prox Cen is of the order of $80 \mathrm{~d}$, its Rossby number is of the order of $R_{\mathrm{O}}=1$ (or $\log R_{\mathrm{O}}=0$ ). This high a Rossby number would put Prox Cen among the stars occupying the very beginning of the linear part of the rotation-activity relation, i.e. among the stars with the lowest measurable activity. From the relation between Rossby number and magnetic flux (Saar 2001; Reiners \& Basri 2008b) one would expect magnetic flux of the order of $100 \mathrm{G}$ for such a small Rossby number. Normalized X-ray activity would be of the order of $\log L_{\mathrm{X}} / L_{\mathrm{bol}}=-5.0$ (Pizzolato et al. 2003; Kiraga \& Stępień 2007), and $\mathrm{H} \alpha$ activity also would be at about $\log L_{\mathrm{H} \alpha} / L_{\mathrm{bol}} \approx-5$ implying that $\mathrm{H} \alpha$ would probably not be detectable as an emission line. The conclusion from the various rotation-activity relations is that from $\mathrm{H} \alpha$ emission, $\mathrm{X}$-ray emission, and from magnetic flux, one would estimate a Rossby number of the order of $\log R_{\mathrm{O}} \approx-0.6$. Together with a convective overturn time of $\tau_{\text {conv }}=70 \ldots 100 \mathrm{~d}$, this leads to a rotation period estimate between $17 \mathrm{~d}$ and $25 \mathrm{~d}$ rather than $80 \mathrm{~d}$. To reach $\log R_{\mathrm{o}} \approx-0.6$ at a rotation period of $80 \mathrm{~d}$, the turnover time needs to be of the order of $300 \mathrm{~d}$ rather than $100 \mathrm{~d}$.

We conclude that Prox Cen is a slow rotator with a rotation period longer than about 15 days, but its activity and magnetic flux are indicative of a rotation period substantially shorter than $80 \mathrm{~d}$. It is less active than more rapid rotators like CN Leo or UV Cet. Its level of chromospheric and coronal activity is relatively high for its moderate magnetic flux or Rossby number, but given the substantial scatter in the relations between these quantities, it lies with other stars on the high activity side of the linear relation between rotation, activity, and magnetic flux in M dwarfs.

Acknowledgements. Based on observations made with the European Southern Observatory telescopes obtained from the ESO/ST-ECF Science Archive Facility. We thank the anonymous referee for a very helpful report. A.R. acknowledges research funding from the DFG as an Emmy Noether fellow under RE 1664/4-1. G.B. acknowledges support from the NSF through grant AST0606748 .

\section{References}

Benedict, G. F., McArthur, B., Nelan, E., et al. 1998, AJ, 116, 429 Bochanski, J. J., West, A. A., Hawley, S. L., \& Covey, K. R. 2007, AJ, 133, 531 Doyle, J. G. 1987, MNRAS, 224, 1

Gilliland, R. L. 1986, ApJ, 300, 339

Guinan, E. F., \& Morgan, N. D. 1996, A\&AS, 1996, 188, 7105

Hawley, S. L., Gizis, J. E., \& Reid, I. N. 1996, AJ, 112, 2799

Hünsch, M., Schmitt, J. H. M. M., Sterzik, M. F., \& Voges, W. 1998, A\&AS, 135,319

Johns-Krull, C., \& Valenti, J. A. 2000, ASPC, 198, 371

Kiraga, M., \& Stępień, K. 2007, AcA, 57, 149

Kunkel, W. E. 1973, ApJS, 213, 25

Kurosawa, R., Harries, T. J., \& Littlefair, S. P. 2006, MNRAS, 372, 1879

Mathioudakis, M., \& Doyle, J. G. 1991, A\&A, 244, 409

Mohanty, S., \& Basri, G. 2003, ApJ, 583, 451

Patten, B. M. 1994, IBVS, 4048, 1

Pizzolato, N., Maggio, A., Micela, G., Sciortino, S., \& Ventura, P. 2003, A\&A, 397,147

Reiners, A. 2007, A\&A, 467, 259

Reiners, A., \& Basri, G. 2006, ApJ, 644, 497

Reiners, A., \& Basri, G. 2007, ApJ, 656, 1121

Reiners, A., \& Basri, G. 2008a, ApJ, in press, [arXiv:0805. 1059]

Reiners, A., \& Basri, G. 2008b, submitted

Saar, S. H. 2001, ASP Conf. Ser., 223, 292

Schmitt, J. H. M. M., \& Liefke, C., A\&A, 417, 651

Ségransan, D., Kervella, P., Forveille, T., \& Queloz, D. 2003, A\&A, 397, L5

Thackeray, A. D. 1950, MNSSA, 9, 9

Walker, A. R. 1981, MNRAS, 195, 1029

West, A. A., Hawley, S. L., Walkowicz, L. M., et al. 2004, AJ, 128, 426 